\title{
Performance Study of CAM over IEEE 802.11p for Cooperative Adaptive Cruise Control
}

\author{
Oyunchimeg Shagdar*§, Fawzi Nashashibi ${ }^{\S}$, and Samir Tohme* \\ Email: oyunchimeg.shagdar@vedecom.fr,fawzi.nashashibi@inria.fr, and samir.tohme@vedecom.fr \\ * Institute VEDECOM, 77 rue des Chantiers, 78000 Versailles, FRANCE \\ $\S$ RITS Project-Team, INRIA Paris, 2 rue Simone Iff, 75589 Paris Cedex 12, FRANCE
}

\begin{abstract}
The IEEE 802.11p is the de-facto vehicular radio communication technology for road safety and efficiency applications. With the advancements in the autonomous vehicle technology, studies on applicability of the IEEE 802.11p and the related protocols for the autonomous driving applications are needed. In this paper, we study the impacts of vehicular communication on platooning control considering that the ETSIstandardised message set Cooperative Awareness Message (CAM) and the IEEE 802.11p are used for both the platooning and cooperative awareness applications. We first develop a theoretical model for the probability of a successful CAM transmission over IEEE 802.11p between platoon members by taking account of the existence of non-platoon vehicles on the road. The model is verified by comparing against simulation results obtained from the NS3 simulator. Finally, we investigate the impacts of the communication performance on the behaviour of platoons, specially the chain stability, when hundreds of vehicles share the wireless channel. The theoretical model reveals that thanks to the capture effect, communications between platoon members drastically outperform communications between arbitrary two vehicles on the road. The simulation results show that in contrast to an adaptive cruise control (ACC), which does not use vehicular communication, the IEEE 802.11p based vehicle to vehicle (V2V) communication aids for realizing stable platoons in highway scenarios.
\end{abstract}

\section{INTRODUCTION}

One of the important applications of Intelligent Transportation Systems (ITS) is platooning, where vehicles drive in an autonomous mode forming a train of vehicles, where each vehicle follows the preceding vehicle maintaining a small inter-vehicle space. A great improvement on traffic flow is expected with such a platooning control especially by the constant spacing policy, i.e., the desired inter-vehicle space is as small as few meters regardless of the velocity [1]. Traditionally, platooning control is achieved based on vehicle embedded sensors such as radars. The sensors are used at each platoon member to measure the distance to its preceding vehicle. Unfortunately such a sensor-only-based approach, called adaptive cruise control (ACC), suffers from the chain instability problem, where the oscillations due to speed changes are not only propagated but also amplified towards the tail of the platoon. The problem is serious specially when the constant spacing policy is applied. The studies show that the problem can be alleviated if not eliminated, when vehicles communicate with each other allowing vehicles to have information on not only the kinematic status of the other vehicles but also their near-future intentions. Communication added ACC, Cooperative ACC (CACC), hence allows vehicles to anticipate the potential problems, enabling them to drive safer and smoother at short inter-vehicle distances [1], [2].
For improved road safety and efficiency, the IEEE is standardised the $802.11 \mathrm{p}$ technology that allows vehicles communicate with each other over the $5.9 \mathrm{GHz}$ radio spectrum [3]. The ETSI adopted the standard under the name of ITS G5 for European usages. In Europe, five $10 \mathrm{MHz}$ channels are allocated for vehicular communication, among one is called control channel $(\mathrm{CCH})$ dedicated especially to road safety applications. Several types of message sets are defined for road safety and efficiency applications; most importantly, Cooperative Awareness Messages (CAM) and Decentralised Environmental Notification Messages (DENM). CAM [4] is periodically broadcasted by each vehicle containing the vehicle's kinematic data, the attribute (vehicle length, height), the movement data (historical and future prediction of path). The primary usage of CAM is for cooperative awareness that allows each vehicle to know the existence and the status of the other vehicles in the vicinity so that potential accidents can be avoided. A DENM is, on the other hand, transmitted upon a detection of an event e.g., an emergency brake and it can be forwarded over multiple hops if necessary.

In contrast to the voluminous literature on vehicular communications dedicated to the road safety applications, very few efforts are made on communications for CACC. Xu et al. [5] studied the information sets necessary for adaptive cruise controlled highway systems and concluded that the location based broadcast and event-triggered broadcast are very useful for autonomous driving applications, while unicast communication is unavoidable for certain applications. Vinel et al. [6] stated that CAMs can serve for the controller at each platoon member and also for the leader for its monitoring activities. ETSI is currently conducting a pre-standardisation study on the CACC use cases and suggesting to extend the CAM contents with detailed information, such as target time gap, for CACC [7]. Obviously, CAMs are still used for cooperative awareness allowing platoons to avoid potential collisions with other vehicles on the road. In this paper, we are interested in studying the impacts of the performances of CAM over the IEEE 802.11 p on platoon behaviors, especially the string stability [8]. In order to do so, we first build a theoretical model of the probability of successful transmission of a CAM over IEEE $802.11 \mathrm{p}$ for intra-platoon communications by taking account of the capture effect in a highway scenario, where normal (human-driven) vehicles also present. Then we validate the model by comparing against simulation results obtained from NS3 [9]. Finally, we integrate our theoretical model into the SIMULINK simulator and evaluate the impacts of CAM over the IEEE $802.11 \mathrm{p}$ on the stability of a platoon targeting a highway scenario with hundreds of vehicles. Specifically, we 
evaluate the chain stability of the communication-aided platoon (CACC) and compare it against that of ACC. The simulation results show that in contrast to the ACC system, which suffers from chain instability problem, the IEEE 802.11 p aided CACC is mostly string stable even when hundreds of vehicles share the wireless channel for transmissions of CAMs.

The paper proceeds as follows. Section II introduces the related work, section III develops an analytical model of the probability of successful transmission of CAM over the IEEE 802.11 system for communication between platoon members. The model is validated in section IV. The stability performances of platoons with and without V2V communication are evaluated and compared in section V. Finally, section VI concludes the paper.

\section{RELATED WORK}

The advantages of CACC over ACC and manually driving vehicles are well studied in the past few years. VanderWerf et al. [10] studied the effects on capacity of increasing market penetration of ACC and CACC vehicles, relative to manually driven vehicles using microscopic traffic simulation. The road capacities are estimated 2050, 2200, and 4500 vehicles per hour for manual driving, ACC, and CACC cases, respectively, when the market penetration is $100 \%$ for the ACC and CACC cases. In [2], Arem et al. studied the impact of CACC on traffic-flow characteristics using a stochastic simulation model. Targeting a highway-merging scenario from four to three lanes, the authors showed that the traffic-flow stability is drastically increased when more CACC-equipped vehicles exist. The study showed a need of communication protocols that allow vehicles to negotiate for complex driving manoeuvres, such as enlarging the inter-vehicle gap for vehicle merging. While the above mentioned studies show the benefits of vehicular communication in ACC systems, the work assumed that the communication is perfect with no information loss and no delay.

The authors of [8] studied the impact of communication delay on longitudinal control. Assuming time division with token based multiple access, the effect of communication in the lead and the preceding vehicles' information are analysed when controllers are triggered by the preceding vehicle information or lead vehicle information. The study showed that a platoon is string stable if all vehicles update their controllers at the same time. Balador et al. [11] proposed to extend the CSMA/CA, the underlying channel access scheme of the IEEE 802.11 p, by token-passing method dedicated to platoon applications. Time-divistion multiple access (TDMA) approach is also used for intra-platoon communications in [12], where Fernandes et al. presented an extended SUMO traffic simulator with a constant spacing platooning model. Thanks to its rather deterministic behaviour, TDMA is indeed an interesting choice for applications with strict requirements. However compared to Carrier Sense Multiple Access with Collision Avoidance (CSMA/CA), which is the underlying channel access method of the IEEE 802.11 systems, TDMA is extremely prone to the network dynamics (e.g., nodes' appear and disappear). Vinel et al [6] provided a detailed description of the contents included in a CAM, and concluded that CAM can be used for platooning control and also help the leader to monitor the platoon members. Targeting the latter usage, the authors conducted a simulation based study on data-age performances of CAM over the IEEE 802.11p. While the ETSI standardised CAM and DENM packets are expected to be transmitted on the $\mathrm{CCH}$ of the $5.9 \mathrm{GHz}$ band, Bohm et al. [13], [14] proposed to use a dedicated service channel for the platoon application. In [14], the authors introduced a channel access method that is combined from contention based channel period dedicated to DENMs and contention free channel access dedicated to CAMs between platoon members in the service channel. We conclude that there is no work yet that studied the impacts of the IEEE 802.11 p on the platoon behaviours. Motivated by this, in this paper, we study the impacts of CAM over IEEE 802.11 p on platoon behaviours, specially the platoon stability, and compare the results against those of an ACC system.

The performances of the IEEE 802.11 p based communication is mainly defined by the signal propagation characteristics and the behaviour of the medium access control (MAC) protocol. Several path loss models are developed to approximate radio signal quality in wireless channels [15]; a number of field tests have been carried out to study the applicability of the models to vehicular environments [16][19]. It has been shown that in highway environments, a simple free-way propagation model is suitable in short inter-vehicle distances and the two-ray interference model is suitable for moderate or long distances [17]. The two-ray interference model however requires high computational cost, and hence the two-ray ground model is often used [15]. As a result, the dual slope linear model should be used since it provides different attenuation factors for the near and far distances [18]. A large number of theoretical models of the IEEE 802.11 MAC protocols have been developed to formulate e.g., the packet loss, throughput, delay performances [20]-[24]. Bianchi [20] conducted the pioneering work, in which a two dimensional Markov chain is built to represent the channel access procedure for Distributed Coordination Function (DCF) for saturated IEEE 802.11 nodes (the transmission queues always contain packets to send). Daneshgran et al. [21] extended the work for throughput study in unsaturated system by taking account of the capture effect. Ma et al. [22] presented a backoff model of DCF in order to analyse the broadcast saturation performances. Huang et al. [23] modeled the Enhanced Distributed Coordination Function (EDCA) of IEEE 802.11e for saturated nodes by taking account of internal collisions. Han et al. [24] studied EDCA for IEEE 802.11p with four access categories (ACs) by also taking account of internal collisions. In this paper, we develop yet another model dedicated to CAM transmissions over the IEEE 802.11p between platoon members considering that normal vehicles (human driven vehicles) also exist on the road. As it will be detailed in the following section, the key differences of the presented model from the previous models are that the model considers of the immediate channel access and post-backoff procedures at non-saturated and saturated nodes by taking account of the capture effect.

\section{SUCCESS PROBABILITY OF CAM TRANSMISSION OVER IEEE 802.11P FOR INTRA-PLATOON COMMUNICATION}

In wireless mobile networks, successful reception of a packet depends on many factors including multi-path fading, Doppler shift, hidden terminal problem, exposed terminal problem, and capture effect. In this paper we are interested in the scenario illustrated in Fig. 1: a platoon of vehicles 


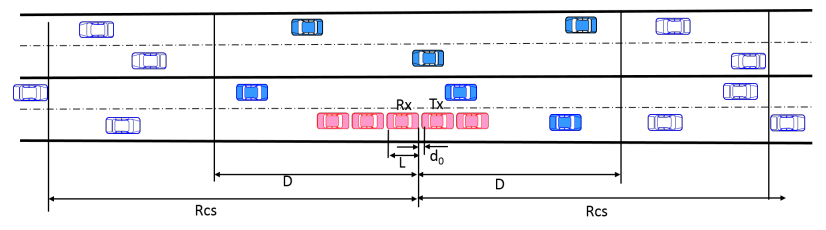

Fig. 1. The target highway scenario, in which a platoon of autonomous vehicles (red vehicles) are sharing the road with normal vehicles (human driven vehicles: blue vehicles)

(red vehicles) is driving on a highway, where normal vehicles (human-driven vehicles) also exist. We consider a CACC, which requires each platoon member to receive CAMs from its preceding vehicle in order to obtain the information on its desired speed. In this case, the intended transmitters and receivers are platoon members, hence the relative speed is close to zero. This allows us to ignore the Doppler shift, which occurs only at a very high relative speed. We also neglect the hidden and exposed terminal problems in the interest of mathematical tractability. Actually, since the intervehicle distance between the intended transmitter and receiver is only few meters, the geographical zone where to have hidden terminals is very small (in line-of-sight cases), the problem of the hidden terminal should indeed be small. The capture effect, i.e., the ability of correctly receiving the intended signal in the presence of interference, however cannot be ignored since the receive signal power from the preceding vehicle in the platoon can be significantly higher than the power of interfering signals arriving from arbitrary vehicles on the road.

Therefore, we can say that a CAM packet transmitted by a vehicle in the platoon is successfully received at the follower if the packet is not collided or the packet is captured at the receiver thanks to a sufficiently high SINR (signal to interference plus noise ratio). Hence letting $P_{n o n-c o l l}$ be the non-collision probability and $P_{c a p}$ be the capture probability, the probability of a successful transmission is

$$
P s=P_{\text {non-coll }}+P_{\text {cap }} .
$$

If the platoon consists of $n_{p}$ vehicles with the bumper to bumper distance $d_{0}$, (see Fig. 1), the platoon occupies $l_{p}=\left(n_{p}-1\right) d_{0}+n_{p} L$ length of the road lane. Here $L$ is the vehicle length. We assume that all other vehicles on the road are normal vehicles, which do not form platoons, and characterised with the Poisson distribution with the density $\beta$ (cars/meters/lane). Thus, the probability of finding $i$ normal vehicles in the $r$ length of a lane is

$$
P(i, r)=\frac{(\beta r)^{i} e^{-\beta r}}{i !},
$$

Since the non-collision probability refers the case when the channel is accessed by only the desired transmitter in the receiver's sensing range (Rcs), letting $\tau$ be the channel access probability, $P_{n o n-c o l l}$ is

$$
P_{\text {non-coll }}=(1-\tau)^{n_{p}-1} \times \sum_{i=0}^{\infty} P(i, R)(1-\tau)^{i},
$$

Here $R=2 s R_{c s}-l_{p}$ is the length of the road occupied by normal vehicles within the sensing range, where $s$ is the number of lanes.
Several studies are made to model capture probability in CSMA/CA systems [21], [25]; the authors of [25] are compared the performances of such methods and showed that the best performing method considers that capture occurs if and only if the power of the desired packet is greater than each of the powers of the joint interfering packets by a factor $z$, i.e.,

$$
p w r_{0}>z \max \left(p w r_{1}, p w r_{2}, \ldots, p w r_{m}\right)
$$

The receive power from a transmitter at a distance $r$ can be expressed

$$
p w r=A r^{\alpha} \text { Ptx }
$$

Here Ptx is the transmission power, $A r^{\alpha}$ is the path loss, where $\alpha$ is the path-loss exponent. For the platooning control, which needs bumper to bumper communication, the distance between the transmitter and receiver is only $d_{0}+L$, hence the desired power is $p w r_{0}=A\left(d_{0}+L\right)^{\alpha}$ Ptx. From (5) and (4), we can find that capture occurs if none of the vehicles within the range of $D$ from the intended receiver transmits (see Fig.1) and at least one simultaneous transmission occurs from outside of the range $D$ (see Fig. 1). Here $D$ is

$$
D=z^{1 / \alpha} \times\left(d_{0}+L\right) .
$$

Hence the capture probability is

Pcap $=(1-\tau)^{n_{p}-1} \sum_{i=1}^{n_{n}} P\left(i, r_{f}\right) \times\left(1-(1-\tau)^{i}\right) \times(1-\tau)^{n_{n}-i}$,

where $n_{n}$ is the number of normal vehicles in the sensing range and $r_{f}=2 s\left(R_{c s}-D\right)$ is the far zone, from where at least one simultaneous transmission must occur. Note that as mentioned earlier, the hidden terminal effect is neglected in this work, hence capture effect takes account of synchronous simultaneous transmissions. By substituting (3) and (7) to (1), the probability of successful transmission between the platoon members is

$$
\begin{aligned}
P s & =(1-\tau)^{n_{p}-1} \\
& \times \sum_{i=0}^{\infty} P(i, R)\left[(1-\tau)^{i}+\sum_{j=1}^{i} P\left(j, r_{f}\right)\left(1-(1-\tau)^{j}\right)(1-\tau)^{i-j}\right]
\end{aligned}
$$

As mentioned earlier, a large amount theoretical models, especially Markov-chain models, presented in the literature, which are to allow finding the channel access probability, $\tau$, in Wi-Fi systems. In what follows, we do present yet another Markov-chain model illustrated in Fig. 2. The model is dedicated to transmissions of CAM packets in the IEEE 802.11p system, whose MAC follows the enhanced distributed channel access (EDCA) mechanism. In contrast to Distributed Coordination Function (DCF) of the IEEE 802.11 legacy MAC, EDCA defines four access categories (ACs) to provide differentiated channel access. The differentiation between ACs is provided by assigning different access parameters (the arbitration inter-frame space, $A I F S$, and the minimum and maximum contention windows, $C W_{\min }$ and $C W_{\max }$ ) to the different ACs. Another important difference of EDCA from DCF is that the backoff counter is decremented at the start of slot time (while in the DCF, the backoff counter is decremented at the end of an idle slot) [24]. Since we are interested in the case where each vehicle sends CAMs, which belong to a same $\mathrm{AC}$, the $\mathrm{AC}$ differentiation does not impact. The key 


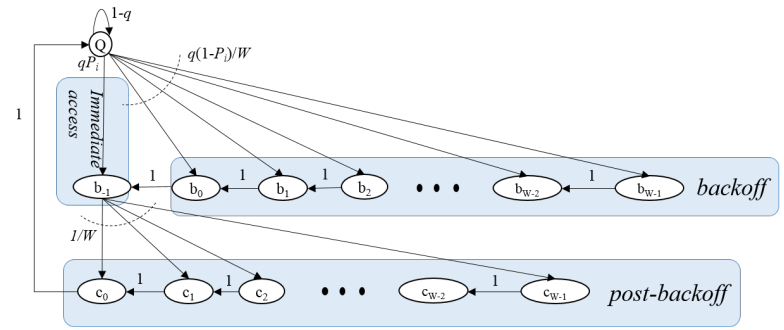

Fig. 2. Markov chain for a broadcast packet transmission in the IEEE $802.11 \mathrm{p}$

differences of the presented model from the existing models are as follows.

- In contrast to the models, which assume that transmission queues at individual nodes are always not empty [20], [22]-[24], the current model considers that the queue can be either empty or not empty. Indeed, when the highway is in an normal situation, we may expect only CAM packets being broadcasted by each vehicle every e.g., $100 \mathrm{~ms}$. In this case, it is difficult to assume that the nodes will always have packets in their transmission queues. The queue state $(Q)$ in Fig. 2 is to capture the possibility of having an empty queue.

- Many existing models ignore the immediate channel access and/or the post-backoff procedures [20]-[24]. The immediate channel access procedure allows the node to transmit without invoking backoff, when the node got a packet to send and the channel is sensed idle during the AIFS period. The post-backoff procedure is taken by all the nodes after a transmission of a frame. The impacts of these procedures are maybe small for a transmission of a unicast packet, which is subject to multiple exponential backoffs. However, the impacts should not be ignored for transmissions of broadcast packets, which are not retransmitted.

As illustrated in Fig. 2, the Markov chain consists of a queue state, $Q, W$ backoff states $b_{j}, W$ post-backoff states $c_{j}$, and a transmission state $b_{-1}$. Here $W$ is $C W_{\min }+1$, where $C W_{\min }$ is the minimum contention window size [26]. Since after the backoff counter is decreased to zero the node has to wait an extra idle slot before transmitting, the current model has separate states for the backoff state $b_{0}$ and transmission state $b_{-1}$. Letting $q$ be the probability of having a packet to send and $p_{i}$ is the probability of the channel being idle, the state transitions of the Markov model are

$$
\begin{array}{cl}
P\{Q \mid Q\}=1-q & \\
P\left\{b_{-1} \mid Q\right\}=q p_{i} & \\
P\left\{b_{j} \mid Q\right\}=\frac{q}{W}\left(1-p_{i}\right), & 0 \leq j \leq W-1 \\
P\left\{b_{j-1} \mid b_{j}\right\}=1, & 0 \leq j \leq W-1 \\
P\left\{c_{j-1} \mid c_{j}\right\}=1 & 0<j \leq W-1 \\
P\left\{c_{j} \mid b_{-1}\right\}=\frac{1}{W}, & 0 \leq j \leq W-1 \\
P\left\{Q \mid c_{0}\right\}=1 &
\end{array}
$$

Eq. (9.a) indicates that if there is no packet to send, $Q$ state persists with the probability of $1-q$. If there is a packet to send (with the probability of $q$ ), a state transition from $Q$ to $b_{i}$ $(i \forall[-1, W-1])$ happens, taking either the immediate channel access or the backoff procedure. More precisely, the frame can be transmitted immediately if the channel was idle during the AIFS period (9.b), otherwise backoff counter is set to a random period of time taken from the range $[0, W-1]$ slots (9.c). To be precise, in the latter case, when the channel becomes idle, the station has to verify if the channel stays idle during the AIFS period and decrements the backoff counter at the end of AIFS. Since the nodes have packets belonging to the same AC, the channel won't become busy during an AIFS, hence the backoff counter is decremented with the probability 1 (9.d-e) [23], [24]. When the packet is transmitted, the post-backoff procedure is taken regardless of the channel and the queue states (9.f). Finally, when the post-backoff counter reaches zero, the state transition from $c_{0}$ to $Q$ happens without any condition $(9 . \mathrm{g})$.

By solving the Markov chain, i.e., $Q+\sum_{i=-1}^{W-1} b_{i}+$ $\sum_{i=0}^{W-1} c_{i}=1$, the channel access probability is found:

$$
\tau=b_{-1}=\left[\frac{1}{q}+1+\frac{(W-1)\left(2-p_{i}\right)}{2 p_{i}}\right]^{-1} .
$$

Assuming that packets are generated following the Poisson process, the probability of having a packet to transmit is

$$
q=1-e^{-\lambda Y_{s}} .
$$

Here $\lambda$ is the packet generation rate and $Y_{s}$ is the average channel service time. Letting $T$ be the time required for a transmission of a frame, $Y_{s}$ is

$$
Y_{s}=\left(1-p_{i}\right)(T+A I F S)+p_{i} \sigma .
$$

Here $\sigma$ is the slot time [26]. Finally, the channel is idle if none of the nodes in the carrier sensing range of the transmitter accesses to the channel:

$$
p_{i}=(1-\tau)^{N},
$$

where $N=n_{p}+n_{n}$, and the probability of having $n_{n}$ normal vehicles in the carrier sensing range can be found by (2). It should be noted that since the distance between the consecutive platoon members is very small (few meters), the sensing range of the transmitter and that of the receiver can be considered as overlapping, and hence $n_{n}$ is same as that in Eq. (7). Eqs. (10)-(13) and Eq. (8) allow us to calculate the probability of a successful transmission between platoon members.

\section{Model Verification: Performance EVAluation OF CAM OVER THE IEEE 802.11P FOR A PLATOON}

In this section, we validate our model by comparing its results to simulation results obtained from the network simulator NS3 version 21 (NS3.21) [9]. The simulator contains the implementations of the TCP/IP stack, including the IEEE 802.11p protocol [27]. Hence, while the theoretical results are found by solving equations Eqs. (10)-(13) and Eq. (8), the simulation results are obtaining by simulating wireless communication among nodes (equipped with the protocol stack, antennas, and etc.) distributed on a highway topology in the simulation environment. The simulation parameters are listed in Table I.

In the simulations, a platoon of five vehicles are driving at 4 meters of inter-vehicle distances on a 4-lane highway. The length of highway is 1000 meters and the width of each lane is 3 meters. On the same highway, normal vehicles are driving at 
TABLE I. SIMULATION PARAMETERS.

\begin{tabular}{c|c}
\hline Road model & Highway (1000 m, 4 lanes) \\
\hline Platoon size & $5 \mathrm{cars}$ \\
\hline Vehicle length & $5 \mathrm{~m}$ \\
\hline Bumper to bumper distance between platoon members & $4 \mathrm{~m}$ \\
\hline CAM transmission rate & $10 \mathrm{~Hz}$ \\
\hline CAM Size & $400 \mathrm{~B}$ \\
\hline Access Technology & ITS G5 \\
\hline Tx power & $23 \mathrm{dBm}$ \\
\hline Carrier sense threshold & $-95 \mathrm{dBm}$ \\
\hline Modulation scheme & QPSK 1/2 $(6 \mathrm{Mbps})$ \\
\hline Antenna pattern & Omnidirectional, gain $=1 \mathrm{dBi}$ \\
\hline Fading model & LogDistance, exponent 2 \\
\hline$C W_{m i n}$ & 15 \\
\hline AIFSN & 6 \\
\hline Slot time $(\sigma)$ & $13 \mu \mathrm{s}$ \\
\hline SIFS & $32 \mu \mathrm{s}$
\end{tabular}

Platoon (simulation) $\llcorner-\mathbb{D - 1}$ Normal vehicles (simulation)) $\llcorner-\mathrm{d}--1$ Success probability (analysis) Non-collision probability (analysis)

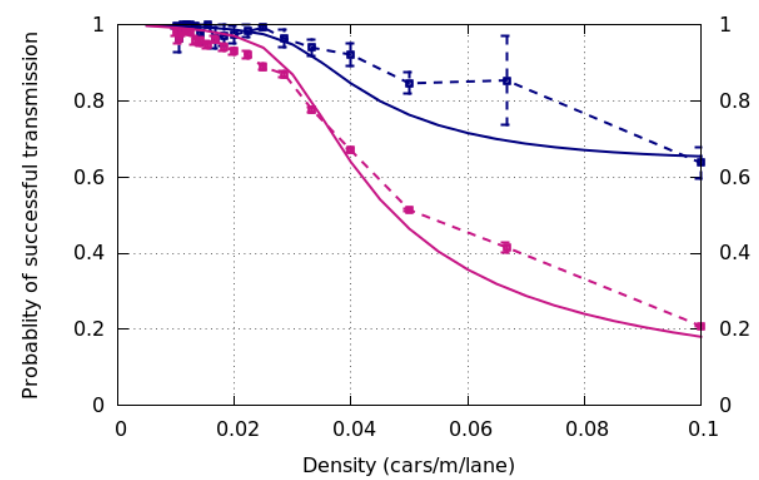

Fig. 3. Comparison of analytical and the simulation results.

density of $\beta$ [cars $/ \mathrm{m} / \mathrm{lane}$. Each vehicle broadcasts 400 Bytes of CAM with the transmission power of $23 \mathrm{dBm}$, which are the commonly agreed values for V2V communications [27]. While CAM generation rate can be varied, we are interested in the cases, where the channel load is high, and hence the vehicles broadcast CAMs at $10 \mathrm{~Hz}$ in the simulation. Signal propagation is modelled by the Log distance model with path loss exponent of 2 as suggested in [17], [18], [27] for highway scenarios. Carrier sense threshold is $-95 \mathrm{dBm}$. The parameters that are necessary for the theoretical model including $C W_{\min }$, $\sigma$, and AIFSN take on the default values of the IEEE $802.11 \mathrm{p}$ system listed in Table I [3]. The capture threshold, $z$, is equal to 5 , which is a value derived from the NS3; the same value is used in [25].

In the simulations, we measured the packet delivery ratio (PDR, i.e., the success probability) for CAM packets for platooning and cooperative awareness. PDR for platooning is measured for two consecutive platoon members and PDR for cooperative awareness is measured for all pairs of vehicles, which are not farther than 500 meters from each other. For analytical results, we calculated the success probability, $P s$, and the non-collision probability, $P_{n o n-c o l}($ see (1)).

Figure 3 compares the analytical and simulation results. The horizontal axis is the density of the normal vehicles $\beta$ (car $/ \mathrm{m} / \mathrm{lane}$ ). Note that for each $\beta$, we conducted ten simulation runs. The average values with the $95 \%$ of confidence intervals are depicted in the figure. As can be seen in the figure, the theoretical success probability developed for platooning, $P s$, roughly agrees to PDR of intra-platoon communication obtained from the simulations. On the other hand, the noncollision probability fits to the PDR results calculated for cooperative awareness. These results indicate that the capture probability is very important to consider for communications between neighbouring vehicles, while it and other effects (hidden terminal etc.) have a small impact for a rough estimation of communications between arbitrary two vehicles. First of all, we can say that Fig. 3 confirms the acceptability of the theoretical model. Secondly, we observe that the packet delivery ratio of CAM for cooperative awareness degrades down to $20 \%$ in the dense scenario $(\beta=0.1)$. This result agrees to the previous efforts that reported the channel congestion problem of the IEEE $802.11 \mathrm{p}$ system [27]. On the other hand, the performance of the same CAM messages outperforms significantly for the platooning application: providing $65 \%$ of the success ratio for the dense scenario. This improvement, which is clearly due to the capture effect, is rather encouraging for a usage of the IEEE $802.11 \mathrm{p}$ for intra-platoon communications.

\section{INVESTIGATING IMPACT OF COMMUNICATION ON PLATOON STABILITY}

In this section, we investigate the impact of CAM over the IEEE $802.11 \mathrm{p}$ on the platoon stability. A difficulty in conducting such a study is that, to the best of our knowledge, there is no tool/simulator exist that can simulate both the wireless communication and the vehicle kinematics. A SIMULINK is a well known tool where we can implement vehicle models and study the issues related to vehicle kinematics, including stability. For this reason, we conduct our study by integrating the communication model developed in Section III into a platoon of autonomous vehicles implemented in SIMULINK. The results of this CACC system are compared against those of ACC, where autonomous vehicles do not communicate. The platoon consists of five Inria's Cycab autonomous vehicles [28] that drive following the constant spacing policy. In ACC, the platoon members can measure the distance to their preceding vehicles using its embedded sensors (e.g., laser). In CACC, in addition to the measured distance, the platoon members exchange information using CAM packets, which contain the desired speed. The success probability of a CAM transmission is modeled by the theoretical modeled presented in Section III, considering that non-platoon vehicles also exist on the 4-lane road, broadcasting CAM packets. CAM generation frequency is fixed to $10 \mathrm{~Hz}$ (which is the actual frequency used for platooning experiments at Inria). The density of normal vehicles is $\beta=0.1$ (cars $/ \mathrm{m} / \mathrm{lane}$ ), which results in more than 320 vehicles on the 1000 meters of road. In CACC, it is possible that, due to packet losses, a platoon member may not receive packets during a long period of time, negatively affecting the safety of the system. To avoid such a situation, if a platoon member does not receive a CAM from its preceding vehicle for longer than a $T_{\max }$ period, it switches to the ACC mode until it receives a new CAM. Otherwise, the vehicle uses the information contained in the most recently received CAM. It should be noted that besides information loss, delay is an important performance indicator of communication systems. However, our NS3 simulation results show that the delays of the CAM packets are not longer than few milliseconds. This 


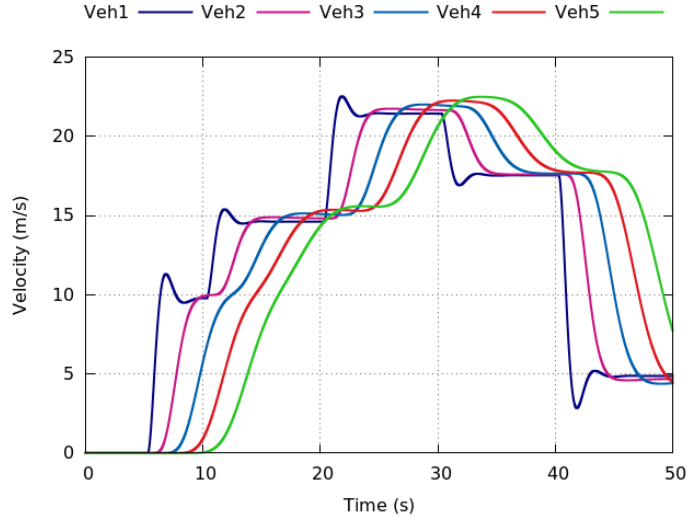

Fig. 4. Velocity of the individual vehicles in ACC.

is much shorter than the CAM generation interval, hence in this paper, we do not consider communication delay.

In the constant spacing policy, each platoon member drives in such a way that the distance to the preceding vehicle is always equal to a desired distance, $d_{i}$. Therefore the range error between platoon member $i-1$ and its follower $i$ can be formulated

$$
\varepsilon_{i}=x_{i-1}-x_{i}-d_{i} .
$$

Here $x_{i}$ is the $i^{\text {th }}$ vehicle's position. Similarly, the range rate error is defined as the speed difference between two consecutive platoon members:

$$
\dot{\varepsilon}_{i}=\dot{x}_{i-1}-\dot{x}_{i} .
$$

To have a stable platoon, it is required that

$$
\varepsilon_{2} \geq \varepsilon_{3} \geq \ldots \geq \varepsilon_{n}
$$

and

$$
\dot{\varepsilon}_{2} \geq \dot{\varepsilon}_{3} \geq \ldots \geq \dot{\varepsilon}_{n},
$$

which refer to the property that the errors do not grow as it propagates towards the tail of the platoon [8]. The requirements of (16) and (17) can be rewritten as:

$$
G_{i}=\varepsilon_{i}-\varepsilon_{i-1} \leq 0
$$

and

$$
g_{i}=\dot{\varepsilon}_{i}-\dot{\varepsilon}_{i-1} \leq 0
$$

Figures 4 and 5 depict the velocities of platoon members in the ACC and CACC systems, respectively. Figure 4 clearly shows that in ACC, each vehicle experiences seconds of delay in adjusting their velocity to that of the preceding vehicle. Conceivably, this is due to the difficulty of controlling its velocity based on only the distance to the preceding vehicle. In comparison, Fig. 5 demonstrates well a benefit of V2V communication for platoon control since the velocity of the preceding vehicle is provided by the wireless communication, which exploits the capture effect (see Fig. 3). It should be mentioned that switching from CACC and ACC was not observed.

Figures 6 and 7 compare the relative range and range rate errors, respectively, for the ACC and CACC systems. Note that following Eqs. (18) and (19), since only positive

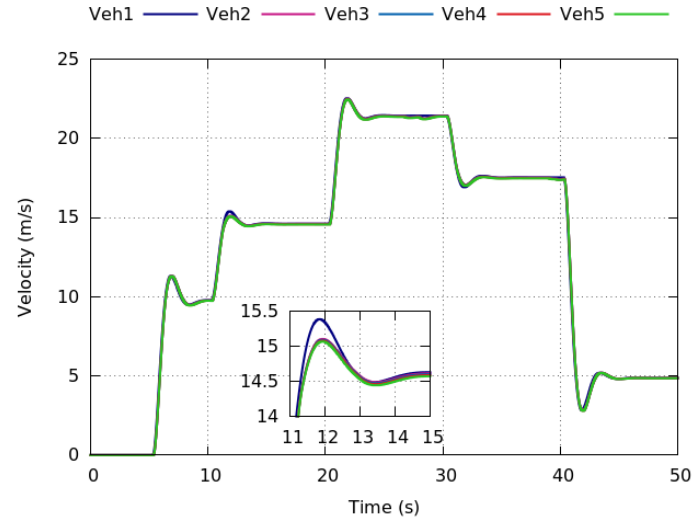

Fig. 5. Velocity of the individual vehicles in CACC

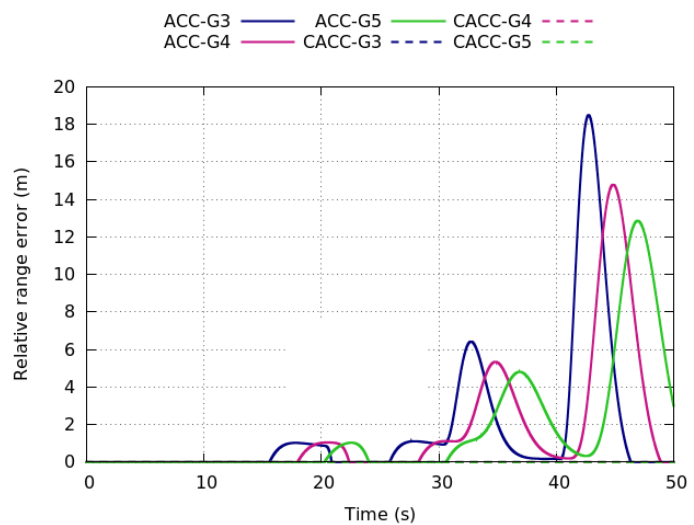

Fig. 6. Comparison of relative range error for the ACC and CACC systems

values indicate unstable chain, the negative values are rounded up to zero (Fig. 6). The obtained range error for CACC was zero. The figures show obvious advantages of the IEEE $802.11 \mathrm{p}$ based the CACC system on the 4-lane highway road. It should be noted however, as Fig. 7 shows, the platoon stability is largely improved by the IEEE $802.11 \mathrm{p}$ communication, however it is not completely stable, i.e., positive $G_{i}$ values are observed, requiring further improvements.

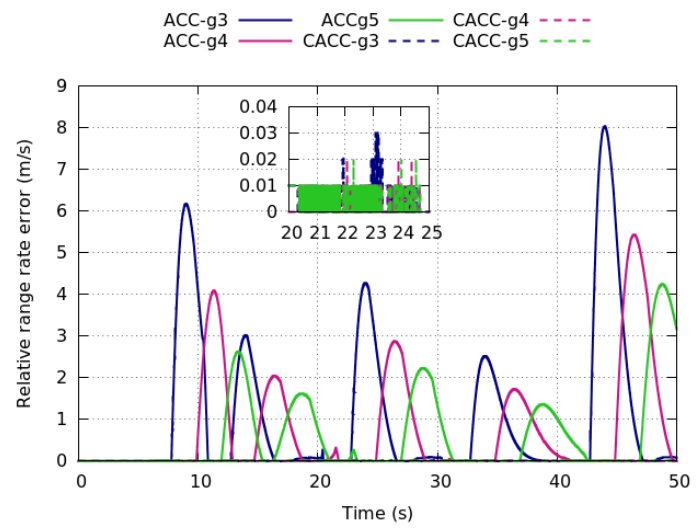

Fig. 7. Comparison of relative range rate error for the ACC and CACC systems 


\section{CONCLUSION}

We studied the applicability of CAM over the IEEE $802.11 \mathrm{p}$ for platooning control. The theoretical model presents that the channel congestion problem largely affects the performance of the communication system for the cooperative awareness application. Specifically, when the road is dense, the probability that a vehicle receives a CAM from another vehicle, which is within 500 meters range can be as low as $20 \%$. On the other hand, thanks to the capture effect, intra-platoon communication can achieve more than $65 \%$ of success probability in the same dense scenario. This is an encouraging result for $\mathrm{CACC}$ or other applications that require communications between neighbouring vehicles. Finally we conducted simulation based study on the impact of CAM over the IEEE $802.11 \mathrm{p}$ on the string stability targeting a highway scenario with hundreds of vehicles. The results show that in contrast to an ACC, which suffers from chain instability problem, the IEEE $802.11 \mathrm{p}$ assisted CACC system presents nearly stable platoon. The future work will include more extensive SIMULINK simulations for different scenarios. Moreover, we will conduct delay analysis of CAM transmission over the IEEE $802.11 \mathrm{p}$ for platooning applications.

\section{REFERENCES}

[1] P. Varaiya, "Smart cars on smart roads: problems of control," IEEE Transactions on automatic control, vol. 38, no. 2, pp. 195-207, 1993.

[2] B. Van Arem, C. J. Van Driel, and R. Visser, "The impact of cooperative adaptive cruise control on traffic-flow characteristics," IEEE Transactions on Intelligent Transportation Systems, vol. 7, no. 4, pp. 429-436, 2006.

[3] IEEE Standard for Information technology - Telecommunications and information exchange between systems - Local and metropolitan area networks - Specific requirement, Part 11: Wireless LAN Medium Access Control (MAC) and Physical Layer (PHY) Specifications, IEEE Computer Society Std., July 2010, iEEE Std 802.11p-2010.

[4] ETSI EN 302 637-2; Intelligent Transport Systems (ITS); Vehicular communications; Basic set of Applications; Part 2: Specification of Cooperative Awareness Basic Service, Std., Nov. 2014, v1.3.2.

[5] Q. Xu, K. Hedrick, R. Sengupta, and J. VanderWerf, "Effects of vehiclevehicle/roadside-vehicle communication on adaptive cruise controlled highway systems," in Vehicular Technology Conference, 2002. Proceedings. VTC 2002-Fall. 2002 IEEE 56th, vol. 2. IEEE, 2002, pp. 12491253.

[6] A. Vinel, L. Lan, and N. Lyamin, "Vehicle-to-vehicle communication in c-acc/platooning scenarios," IEEE Communications Magazine, vol. 53, no. 8, pp. 192-197, 2015.

[7] ETSI TR 103 299; Intelligent Transport Systems (ITS); Cooperative Adaptive Cruise Control (CACC); Pre-standardization study, Std., March 2016, draft V0.0.8

[8] X. Liu, A. Goldsmith, S. Mahal, and J. K. Hedrick, "Effects of communication delay on string stability in vehicle platoons," in Intelligent Transportation Systems, 2001. Proceedings. 2001 IEEE. IEEE, 2001, pp. $625-630$.

[9] "Network Simulator NS-3," 2014. [Online]. Available: http://www. nsnam.org/

[10] J. Vander Werf, S. Shladover, M. Miller, and N. Kourjanskaia, "Effects of adaptive cruise control systems on highway traffic flow capacity," Transportation Research Record: Journal of the Transportation Research Board, no. 1800, pp. 78-84, 2002.

[11] A. Balador, A. Bohm, E. Uhlemann, C. T. Calafate, and J.-C. Cano, "A reliable token-based mac protocol for delay sensitive platooning applications," in Vehicular Technology Conference (VTC Fall), 2015 IEEE 82nd. IEEE, 2015, pp. 1-5.

[12] P. Fernandes and U. Nunes, "Platooning of autonomous vehicles with intervehicle communications in sumo traffic simulator," in Intelligent Transportation Systems (ITSC), 2010 13th International IEEE Conference on. IEEE, 2010, pp. 1313-1318.
[13] A. Böhm and K. Kunert, "Data age based retransmission scheme for reliable control data exchange in platooning applications," in 2015 IEEE International Conference on Communication Workshop (ICCW). IEEE, 2015, pp. 2412-2418.

[14] A. Böhm, M. Jonsson, and E. Uhlemann, "Performance comparison of a platooning application using the ieee $802.11 \mathrm{p}$ mac on the control channel and a centralized mac on a service channel." in WiMob. Citeseer, 2013, pp. 545-552.

[15] A. Goldsmith, Wireless communications. Cambridge university press, 2005.

[16] R. He, A. F. Molisch, F. Tufvesson, Z. Zhong, B. Ai, and T. Zhang, "Vehicle-to-vehicle propagation models with large vehicle obstructions," IEEE Transactions on Intelligent Transportation Systems, vol. 15, no. 5, pp. 2237-2248, 2014.

[17] C. Sommer, S. Joerer, and F. Dressler, "On the applicability of two-ray path loss models for vehicular network simulation." $V N C$, vol. 12, pp. 64-69, 2012.

[18] O. Shagdar, A. Daniel, and S. Primak, "Beacon delivery over practical v2x channels," in ITS Telecommunications (ITST), 2013 13th International Conference on. IEEE, 2013, pp. 68-73.

[19] C. Sommer, D. Eckhoff, R. German, and F. Dressler, "A computationally inexpensive empirical model of ieee $802.11 \mathrm{p}$ radio shadowing in urban environments," in Wireless On-Demand Network Systems and Services (WONS), 2011 Eighth International Conference on. IEEE, 2011, pp. 84-90.

[20] G. Bianchi, "Performance analysis of the ieee 802.11 distributed coordination function," Selected Areas in Communications, IEEE Journal on, vol. 18, no. 3, pp. 535-547, 2000.

[21] F. Daneshgaran, M. Laddomada, F. Mesiti, and M. Mondin, "Unsaturated throughput analysis of ieee 802.11 in presence of non ideal transmission channel and capture effects," Wireless Communications, IEEE Transactions on, vol. 7, no. 4, pp. 1276-1286, 2008.

[22] X. Ma and X. Chen, "Performance analysis of ieee 802.11 broadcast scheme in ad hoc wireless lans," IEEE Transactions on Vehicular Technology, vol. 57, no. 6, pp. 3757-3768, 2008.

[23] C.-L. Huang and W. Liao, "Throughput and delay performance of ieee 802.11 e enhanced distributed channel access (edca) under saturation condition," IEEE Transactions on Wireless Communications, vol. 6 , no. 1, pp. 136-145, 2007.

[24] C. Han, M. Dianati, R. Tafazolli, R. Kernchen, and X. Shen, "Analytical study of the ieee $802.11 \mathrm{p}$ mac sublayer in vehicular networks," IEEE Transactions on Intelligent Transportation Systems, vol. 13, no. 2, pp. 873-886, 2012.

[25] J. Y. Zhao and O. C. Ugweje, "Analysis of capture probability performance techniques for wireless lan," in Vehicular Technology Conference, 2002. VTC Spring 2002. IEEE 55th, vol. 3. IEEE, 2002, pp. $1190-1194$.

[26] IEEE 802.11 Working Group, "IEEE Standard for Information Technology - Telecommunications and information exchange between systems - Local and metropolitan area networks -Specific requirements - Part 11: Wireless LAN Medium Access Control (MAC) and Physical Layer (PHY) specifications Amendment 8: Medium Access Control (MAC) Quality of Service Enhancements," November 2005.

[27] ETSI TR 101 612; Intelligent Transport Systems (ITS); Cross Layer DCC Management Entity for operation in the ITS G5A and ITS G5B medium; Report on Cross layer DCC algorithms and performance evaluation, Std., Sep. 2014, v1.1.1.

[28] F. Navas, V. Milanés, and F. Nashashibi, "Using Plug\&Play Control for stable ACC-CACC system transitions," in Intelligent Vehicles Symposium 2016, Gothemburg, Sweden, Jun. 2016. [Online]. Available: https://hal.inria.fr/hal-01304542 